\title{
CONTINUOUS COLLECTIONS OF DECOMPOSABLE CONTINUA ON A SPHERICAL SURFACE
}

\author{
ELDON DYER
}

In this paper a study is made of continuous collections of decomposable continua on a spherical surface. Properties of the decomposition spaces of such collections filling up continua are established, and a characterization of the decomposition spaces of such collections filling up a spherical surface is obtained.

The results of the present paper are related to certain results obtained by R. D. Anderson. He has shown [2] that there is a continuous collection of nondegenerate continua filling up the plane which is with respect to its elements homeomorphic to the plane, and that there is a continuous collection of continua filling up a plane onedimensional continuous curve which is with respect to its elements homeomorphic to the plane. On the other hand, he has obtained [1] a characterization of the plane decomposition spaces of continuous collections of nondegenerate continuous curves filling up a plane continuum. Such decomposition spaces are special types of hereditary continuous curves. The results of the present paper show that the decomposition spaces of continuous collections of decomposable continua filling up compact plane continua are special types of hereditary continuous curves, and consequently that even under this weaker hypothesis such collections are dimension reducing if they fill up twodimensional continua and are dimension preserving if they fill up one-dimensional continua. It might be noted that there do exist such collections filling up one-dimensional continua, but that Theorem 2 of this paper gives a strong restriction about the nature of such collections.

Theorems about continuous collections of continua in a compact metric space can be stated equivalently in terms of monotone interior transformations (p. 130 of reference [10]).

It is understood throughout this paper that space is compact and metric.

Definitions. If $H$ is a point set and $\epsilon$ is a positive number, $V(\epsilon, H)$ denotes the set of all points $P$ such that some point of $H$ is at a distance from $P$ of less than $\epsilon$. If $H$ and $K$ are point sets, $d(H, K)$ will be used to denote the greatest lower bound of the set of all positive numbers $\epsilon$ such that $K$ intersects $V(\epsilon, H)$. For two

Presented to the Society, April 24, 1954; received by the editors May 29, 1954. 
such sets, $S(H, K)$ is the greatest lower bound of the set of all positive numbers $\epsilon$ such that $K$ lies in $V(\epsilon, H)$ and $H$ lies in $V(\epsilon, K)$.

If the capitalized letter $R$ denotes a collection of closed point sets, the corresponding capitalized German letter $\Re$ denotes the decomposition space in which the points are the elements of $R$ and if $p$ and $q$ are points of $\Re$, the distance between $p$ and $q$ is $S(p, q) . R^{*}$ denotes the sum of all the elements of $R$.

The following lemma is well known and no argument is given for it here:

LemMA 1. If $R$ is a collection of closed and compact point sets in a completely separable, metric space $T$, then $\Re$ is a completely separable, metric space.

Definitions. Let $G$ denote a continuous collection of mutually exclusive continua.

If $k$ is a subcontinuum of an element $g$ of $G$ and $k$ is not a subset of the closure of $g-k$, then $m(k)$ denotes the least number such that no point of $k$ is at a distance more than that number from $g-k$. If $k$ is such a subcontinuum of an element $g$ of $G, \epsilon$ is a positive number which is not greater than $m(k)$ and $z$ is the set of all points of $k$ at a distance from $g-k$ of not less than $\epsilon, M(\epsilon, k)$ denotes the least upper bound of the set of all numbers $e$ such that there exist a point $P$ of $z$ and sequences $g_{1}, g_{2}, \cdots, a_{1}, a_{2}, \cdots, b_{1}, b_{2}, \cdots$ and $e_{1}, e_{2}, \cdots$ such that, for each positive integer $n, e_{n}$ is a positive number and $a_{n}$ and $b_{n}$ are points of the element $g_{n}$ of $G$ and each subcontinuum of $g_{n}$ containing $a_{n}$ and $b_{n}$ contains a point at a distance from $k$ of not less than $e_{n}$ and the sequence $g_{1}, g_{2}, \cdots$ converges to $g$, the sequences $a_{1}, a_{2}, \cdots$ and $b_{1}, b_{2}, \cdots$ converge to $P$ and $e_{1}, e_{2}, \cdots$ converges to $e$.

If $\epsilon$ is a positive number and $g$ is an element of $G$ containing a subcontinuum $k$ for which $m(k)$ is not less than $\epsilon$, let $C(\epsilon, g)$ denote the least upper bound of the set of all numbers $M(\epsilon, k)$ for all such subcontinua $k$ of $g$. If, for each subcontinuum $k$ of $g, m(k)$ is less than $\epsilon$, let $C(\epsilon, g)$ be zero.

The function $C(\epsilon, g)$ furnishes a measure of the lack of equicontinuity of convergence of those sequences of elements of $G$ which converge to $g$. The following lemma is proved in the first paragraph on p. 592 of reference [3]:

LEMMA 2. If $K$ is a continuous collection of mutually exclusive continua and $\Omega$ is a compact metric continuum, there is a subcollection $H$ of $K$ which is a dense inner limiting set in $\Omega$ such that if $h$ is an element of $H$ and $e$ is a positive number, $C(e, h)$ is zero. 
J. H. Roberts obtained [7] the special case of this lemma in which all of the elements of the collection $K$ are arcs.

Definitions. Throughout the remainder of this paper $S$ will denote a spherical surface in three-dimensional Euclidean space and $G$ will denote a continuous collection of mutually exclusive decomposable continua filling up a continuum on $S$. The metric used is the ordinary distance between points. If $C$ is a circle on $S$ which is not a great circle, the center of $C$ is that point $P$ of $S$ which is equidistant from all points of $C$ and is at a distance from $C$ of less than $2^{1 / 2} \cdot r$, where $r$ is the radius of $S$. The distance from $P$ to $C$ is the radius of $C$. If $P$ is a point of $S, I$ is a closed number interval of positive numbers less than $2^{1 / 2} \cdot r$, and $e$ is a positive number which is not greater than $2 r$, then $C(P, I, e)$ denotes the collection of all circles on $S$ with radius in $I$ and center a point of $S$ at a distance from $P$ of less than $e$. The radius of a domain $D$ on $S$ is the least number $e$ such that if $J$ is a circle on $S$, which is not a great circle, and the complementary domain of $J$ containing its center is a subset of $D$, the radius of $J$ is less than or equal to $e$.

The statement that the point $P$ of continuum $M$ is a local separating point of $M$ means that there is a domain $R$ with respect to $M$ containing $P$ such that if $C$ is the component of $R$ containing $P, R-P$ is the sum of two mutually separated sets each intersecting $C$ (p. 61 of reference $[10])$.

THEOREM 1. If $G^{*}$ is a subset of the boundary of a connected open set lying in $S-G^{*}$, there is a subcollection $R$ of $G$ which is a dense inner limiting set in (S) and each of whose elements is a local separating point of (5).

Proof. This theorem is established by showing that there is a subcollection $N$ of $G$ such that for each element $n$ of $N$, it is possible to define a notion of a side of $n$ so that $n$ has either one or two sides. It is then shown that, speaking roughly, those elements of $G$, lying near $n$, lie in only one side of $n$, and that if there are elements of $G$ in each side of $n$ near $n, n$ is a local separating point of $B$. It is next shown that there is a subcollection $R$ of $N$ such that for each element $r$ of $R$, there are elements of $G$ in each side of $r$ near $r$.

Since $G^{*}$ does not intersect two complementary domains of any element of $G$, not more than countably many elements of $G$ have two complementary domains. There is a subcollection $N$ of $G$, no element of which cuts $S$, such that $\mathfrak{R}$ is a dense inner limiting set in $\mathcal{B}$, and such that if $n$ is an element of $N$ and $e$ is a positive number, $C(e, n)$ is zero. This follows immediately from Lemma 2 . 
Let $n$ denote an element of $N$. Since $n$ is decomposable, it is the sum of two of its proper subcontinua, $n_{1}$ and $n_{2}$. There is an arc $\alpha^{\prime \prime}$, lying except for its end points in $S-n$, which intersects $n_{1}$ at a point $P_{1}$ not in $n_{2}$ and intersects $n_{2}$ at a point $P_{2}$ not in $n_{1}$. By Theorem 34, p. 203 of reference [5], there are just two complementary domains, $D_{1}$ and $D_{2}$, of $n+\alpha^{\prime \prime}$, and $\alpha^{\prime \prime}$ is a subset of the boundary of each of them. Since $C(e, n)$ is zero for every positive number $e$, there is a positive number $e$ such that if $g$ is an element of $G, S(g, n)$ is less than $e$, and $A, B, C$, and $D$ are points of $g$ such that $d\left(A, P_{1}\right), d\left(B, P_{1}\right)$, $d\left(C, P_{2}\right)$, and $d\left(D, P_{2}\right)$ are less than $e$, then there are subcontinua $g_{1}$ and $g_{2}$ of $g, g_{1}$ containing $A$ and $B$ and $g_{2}$ containing $C$ and $D$, such that no point of $g_{1}$ is at a distance from $P_{2}$ of less than $d\left(P_{2}, n_{1}\right) / 2$, and no point of $g_{2}$ is at a distance from $P_{1}$ of less than $d\left(P_{1}, n_{2}\right) / 2$. Let $E$ and $F$ denote points of $\alpha^{\prime \prime}$ between $P_{1}$ and $P_{2}$ such that the subarcs $E P_{1}$ and $F P_{2}$ of $\alpha^{\prime \prime}$ have diameters less than $e$.

Since $G^{*}$ is a subset of the boundary of a connected set lying in its complement, there do not exist a point of that complement and two elements of $G$ each of which separates $n$ from that point. Therefore, there is a positive number $c$ such that if $g$ is an element of $G$ and $S(g, n)$ is less than $c, g$ does not separate $n$ from $E F$ in $S$. Suppose there is an element $g$ of $G$ for which $S(g, n)$ is less than both $d(n$, arc $E F) / 2$ and $c$, which has subcontinua $g_{1}$ and $g_{2}$ which respectively lie in $D_{1}+\alpha^{\prime \prime}$ and $D_{2}+\alpha^{\prime \prime}$ and are irreducible from $E P_{1}$ to $F P_{2}$. If $g_{1}$ and $g_{2}$ have a common point in $E P_{1}$, let $\beta$ denote one such point. If they do not, let $\beta^{\prime}$ denote a subarc of $E P_{1}$ irreducible from $g_{1}$ to $g_{2}$. There is an $\operatorname{arc} \beta$, irreducible from $g_{1}$ to $g_{2}$, having the same end points as $\beta^{\prime}$ and not intersecting $\alpha^{\prime \prime}$, except in points of $\beta^{\prime}$, such that the arc $E P_{1}$ in which $\beta$ is substituted for $\beta^{\prime}$ has diameter less than $e$, and such that if $g$ has a point $Q$ on an open segment of $\beta$, then $Q$ is a limit point of subsets of $g$ from both sides of $\beta$. Let $\alpha^{\prime}$ denote the arc $\alpha^{\prime \prime}$ in which $\beta$ is substituted for $\beta^{\prime}$. Similarly, define arcs (or a point) $\gamma^{\prime}$ and $\gamma$ for $F P_{2}$ and substitute $\gamma$ for $\gamma^{\prime}$ in $\alpha^{\prime}$ to obtain the arc $\alpha$. Let $\Delta=\beta+\gamma+g_{1}+g_{2}$. There exist an arc from $n$ to $g_{1}$ not intersecting $\alpha+g_{2}$ and an arc from $n$ to $g_{2}$ not intersecting $\alpha+g_{1}$. Let $D$ denote the complementary domain of $\Delta$ containing $E F$, and $A B$ denote the irreducible subarc of $\alpha$ from $\Delta$ to $\Delta$ containing $E F$. By Theorem 34, p. 203 of reference [5], $n$ and $E F$ lie in different complementary domains of $\Delta$.

There is an arc $\eta$ from a point of $E F$ to a point of $n$ which does not intersect $g$ and is such that if $Z$ is an irreducible subarc of $\eta$ from $\beta$ to $\beta$ (or from $\gamma$ to $\gamma$ ), both complementary domains of $\beta+Z$ (or $\gamma+Z$ ) intersect $g$, and any two successive components on $\eta$ of $\eta-\eta \cdot(\beta+\gamma)$, such that the subset of $\eta$ between them lies on $\beta$, abut 
on $\beta$ from different sides, and similarly for $\gamma$. In the order from $E F$ to $n$, either $\eta$ intersects $\beta$ before $\gamma$ or $\gamma$ before $\beta$. Suppose $\eta$ intersects $\gamma$ before $\beta$ (the other case can be handled similarly). Let $\zeta$ denote the subarc of $\eta$ from $E F$ to $\gamma$, and $\epsilon$ denote the closure of the next component on $\eta$ of $\eta-\eta \cdot(\beta+\gamma)$. Let $\xi$ denote the component of $\eta \cdot \gamma$ intersecting $\zeta$ and $\epsilon$. $\xi$ is either a point or an arc. There are points $T$ and $Q$ of $g$ on $\gamma$ such that $\xi$ is between them, and no point of $g$ on $\gamma$ is between them. Let $V$ denote the first point of $\xi$ from $T$ to $Q$, and let $U$ denote the first point of $\xi$ from $Q$ to $T$. No point of $\zeta+\epsilon$ not in $\xi$ is between $T$ and $Q$ on $\gamma$. Since $\epsilon$ and $\zeta$ abut on $\gamma$ from different sides, by Theorem 32, p. 201 of reference [5], $T V$ and $U Q$ abut on $\epsilon+\xi+\zeta$ from different sides. There is a subcontinuum $h$ of $g$ containing $T$ and $Q$ such that none of its points is at a distance from $P_{1}$ of less than $d\left(P_{1}, n_{2}\right) / 2$. If $\eta$ intersects $\beta$, there is a point $R$ of $\eta$ at a distance less than $e$ from $P_{1}$ such that the subarc of $\eta$ from $R$ to $E F$ does not intersect $\beta$, and such that there is an $\operatorname{arc} \mu$ from $R$ to $n$ which does not intersect $h+F P_{1}$. Let $\eta^{\prime}$ denote $\eta$ if it does not intersect $\beta$, or a subarc from $E F$ to $n$ of the subarc of $\eta$ from $E F$ to $R$ plus the arc $\mu$, if $\eta$ does intersect $\beta$. TV and $U Q$ abut on $\eta^{\prime}$ from different sides. By Theorem 34(2), p. 203 of reference [5], $T$ and $Q$ lie in different components of $S-\left(\eta^{\prime}+n+F P_{1}\right)$. However, there is a continuum, $h$, containing them that does not intersect $\eta^{\prime}+n+F P_{1}$. This is a contradiction.

If there were a sequence $g_{1}, g_{2}, \cdots$ of continua of $G$ converging to $n$ such that for each positive integer $i, g_{i}$ contained two subcontinua, $g_{i}^{1}$ and $g_{i}^{2}, g_{i}^{1}$ lying in $D_{1}$ and $g_{i}^{2}$ lying in $D_{2}$, such that each of the sequences $g_{1}^{1}, g_{2}^{1}, \cdots$ and $g_{1}^{2}, g_{2}^{2}, \cdots$ converged to a subcontinuum of $n$ containing $P_{1}$ and $P_{2}$, then since $C(e, n)$ is zero for every positive number $e$, there would be a continuum in $G$ which has the characteristics of that in the previous impossible supposition.

Therefore, there is a positive number $d$ such that if $g$ is an element of $G$ and $S(g, n)$ is less than $d$, and $g_{1}$ and $g_{2}$ are subcontinua of $g$, neither intersecting $\alpha$ and each containing a point at a distance from $P_{1}$ of less than $d$ and a point at a distance from $P_{2}$ of less than $d$, then either $g_{1}$ and $g_{2}$ lie in $D_{1}$ or they lie in $D_{2}$. Every element $g$ of $G$ for which $S(g, n)$ is less than $d$ contains a subcontinuum $q$, not intersecting $\alpha$, which contains a point at a distance from $P_{1}$ of less than $d$ and a point at a distance from $P_{2}$ of less than $d$. If $q$ lies in $D_{1}, g$ will be said to lie on the $D_{1}$ side of $n$; if $q$ lies in $D_{2}, g$ will be said to lie on the $D_{2}$ side of $n$. Let $Z_{1}$ denote the set of all elements $z$ of $G$ for which $S(z, n)$ is less than $d$, and which lie on the $D_{1}$ side of $n$; and let $Z_{2}$ denote the similar set for $D_{2} . B_{1}$ and $Z_{2}$ are mutually separated in $\mathbb{B}$, and if $n$ is in both $\overline{3}_{1}$ and $\overline{3}_{2}, n$ is a local separating point of $\mathbb{S}$. 
Suppose there is an uncountable subcollection $M$ of $N$ such that no element of $M$ is a local separating point of $(S)$. For each element $m$ of $M$, let $m_{1}$ and $m_{2}$ denote two proper subcontinua of $m$ whose sum is $m, \alpha_{m}$ denote an arc lying except for its end points in $S-m, P_{1}^{m}$ and $P_{2}^{m}$ denote the two end points of $\alpha_{m}, P_{1}^{m}$ lying in $m_{1}$ but not $m_{2}$, and $P_{2}^{m}$ lying in $m_{2}$ but not $m_{1}$, and $D_{1}^{m}$ and $D_{2}^{m}$ denote the complementary domains of $m+\alpha_{m}$. There is a positive number $d_{m}$ such that if $g$ is an element of $G$ and $S(g, m)$ is less than $d_{m}, g$ lies on either the $D_{1}^{m}$ side of $m$ or the $D_{2}^{m}$ side of $m$. There is a positive number $e_{m}$, less than $d_{m}$, and one of the complementary domains of $m+\alpha_{m}$, say $E_{m}$, such that if $g$ is an element of $G$ and $S(g, m)$ is less than $e_{m}$, then $g$ is on the non- $E_{m}$ side of $m$. It can be shown by an indirect argument that for each element $m$ of $M$, there is a positive number $\delta$ such that if $I$ is a closed interval of positive numbers, each of which is less than $\delta$, there is a positive number $e$ such that if $C_{1}$ and $C_{2}$ are elements of $C\left(P_{1}^{m}, I, e\right)$ and $C\left(P_{2}^{m}, I, e\right)$, respectively, and $g$ is an element of $G$ for which $S(g, m)$ is less than $e$, then a complementary domain of $\mathrm{Cl}\left(m+g+\right.$ int. $C_{1}+$ int. $\left.C_{2}\right)$ lying in $E_{m}$ has in its boundary a subset of $\alpha_{m}$ irreducible from $C_{1}$ to $C_{2}$ and a subcontinuum of $m$ irreducible from $C_{1}$ to $C_{2}$. The following statement is implied by the fact that no element of $M$ separates $S$; its form is for convenience in concluding the statement in the next paragraph. If $m$ is an element of $M$ and $\epsilon$ is a positive number, there is a positive number $\delta$ such that if $I$ is a closed interval of positive numbers each of which is less than $\delta$, there is a positive number $e$ such that if $C_{1}$ and $C_{2}$ are elements of $C\left(P_{1}^{m}, I, e\right)$ and $C\left(P_{2}^{m}, I, e\right)$, respectively, and $g$ is an element of $G$ for which $S(g, m)$ is less than $e$, then if $\mu$ is the set of all subcontinua of $m$ irreducible from $C_{1}$ to $C_{2}$, and $\gamma$ is the set of all subcontinua of $g$ irreducible from $C_{1}$ to $C_{2}$, and $h$ and $k$ are elements of $\mu+\gamma$, not more than one complementary domain of $C_{1}+C_{2}+h+k$ has radius more than $\epsilon$.

For each element $m$ of $M$, there exist a closed number interval $I_{m}$ and a positive number $f_{m}$, which is less than every number in $I_{m}$, such that if $C_{1}$ and $C_{2}$ are elements of $C\left(P_{1}^{m}, I_{m}, f_{m}\right)$ and $C\left(P_{2}^{m}, I_{m}, f_{m}\right)$, respectively, then

(1) $\alpha_{m}$ has only one subarc, $\beta_{m}$, irreducible from $C_{1}$ to $C_{2}$;

(2) there is a subcontinuum of $m$ irreducible from $C_{1}$ to $C_{2}$ and a complementary domain $D_{m}$ of $m+\beta_{m}+C_{1}+C_{2}$ having $\beta_{m}$ and that subcontinuum on its boundary such that if $g$ is an element of $G$ for which $S(g, m)$ is less than $f_{m}$, then no subcontinuum of $g$ which is irreducible from $C_{1}$ to $C_{2}$ intersects $D_{m}$; and

(3) if $g$ is an element of $G$ for which $S(g, m)$ is less than $f_{m}, \mu$ is the set of all subcontinua of $m$ irreducible from $C_{1}$ to $C_{2}, \gamma$ is the set 
of all subcontinua of $g$ irreducible from $C_{1}$ to $C_{2}$, and $h$ and $k$ are elements of $\mu+\gamma$, then not more than one complementary domain of $h+k+C_{1}+C_{2}$ has radius more than half of the radius of $D_{m}$.

By repeated alternate applications of Lemma 1 and the theorem that some point of each uncountable point set in a completely separable, metric space is a condensation point of that set, there exist a closed number interval $I$, a closed number interval $J$, the least number of which is $3 / 4$ 's of the greatest, a positive number $d$, and an uncountable subcollection $M^{\prime}$ of $M$ such that for each element $m$ of $M^{\prime}, I$ is a subinterval of $I_{m}, d$ is less than $f_{m}$, and the radius of $D_{m}$ is in $J$. There are three elements $h, k$, and $m$ of $M^{\prime}$ such that if $x, y$, and $z$ are $h, k$, and $m$, then $S(x, y), d\left(P_{1}^{x}, P_{1}^{\nu}\right)$, and $d\left(P_{2}^{x}, P_{2}^{\nu}\right)$ are less than $d$. Let $C_{1}$ and $C_{2}$ denote circles with radii in $I$ and centers $P_{1}^{h}$ and $P_{2}^{h}$, respectively. $C_{i}$ is in $C\left(P_{i}^{x}, I_{x}, f_{x}\right)$, where $i$ is either 1 or 2 and $x$ is either $h, k$, or $m$. Let $X, Y$, and $Z$ denote the elements of $\mu_{x}, \mu_{y}$, and $\mu_{z}$ on the boundary of $D_{x}, D_{y}$, and $D_{z}$, respectively. Suppose that two of the arcs $\beta_{h}, \beta_{k}$, and $\beta_{m}$ separate one of the continua $X$, $Y$, and $Z$ from one of the other two in $S-\left(C_{1}+C_{2}+\right.$ int. $C_{1}+$ int. $\left.C_{2}\right)$. Then the sum of those two continua, together with $C_{1}+C_{2}$, has two complementary domains, each of radius greater than the least number in $J$, which is greater than one-half of the radius of each of the domains $D_{h}, D_{k}$, and $D_{m}$. This contradicts condition (3). Therefore, two of the continua $X, Y$, and $Z$ separate the third from $\beta_{h}+\beta_{k}+\beta_{m}$ in $S-\left(C_{1}+C_{2}+\right.$ int. $C_{1}+$ int. $\left.C_{2}\right)$. But this contradicts condition (2) for one of the continua $h, k$, or $m$.

Since not more than countably many elements of $N$ fail to be local separating points of $\mathfrak{G}$, if $\Re$ is the set of all points of $\mathfrak{N}$ which are local separating points of $B, \Re$ is a dense inner limiting set in $B$.

Theorem 2. If $a$ and $b$ are elements of $G, \mathfrak{S}$ is an irreducible subcontinuum of \& from $a$ to $b$, and $\alpha$ is an arc in $\left(S-H^{*}\right)+a+b$ which is irreducible from $a$ to $b$, then $H^{*}$ is not a subset of the boundary of any complementary domain of $H^{*}+\alpha$ having $\alpha$ in its boundary.

Proof. Suppose this theorem is false. Then there exist elements $a$ and $b$ of $G$, a subcollection $H$ of $G$, and an arc $\alpha$ as stated in the hypothesis of the theorem for which there is a complementary domain $D$ of $H^{*}+\alpha$ having $H^{*}+\alpha$ as its boundary. By Theorem 1 of this paper and Corollary 9.21 of reference [10], there are two elements $p$ and $q$ of $\dot{H}$ such that $C(e, p)$ and $C(e, q)$ are zero for every positive number $e$, and such that $\mathfrak{E}-(p+q)$ is the sum of two mutually separated sets, one of which contains both $a$ and $b$. Since $\mathfrak{S}$ is an irreducible continuum from $a$ to $b, p$ is a cut point of $\mathfrak{W}$.

The point set $p$ is the sum of two of its proper subcontinua $p_{1}$ 
and $p_{2}$. Let $A$ and $B$ denote the end points of $\alpha$ on $a$ and $b$, respectively. There exist a point $P_{1}$ of $p_{1}$ but not in $p_{2}$ and a point $P_{2}$ of $p_{2}$ but not in $p_{1}$. By an argument like that on p. 592 of reference [3], there are subcontinua $h_{1 A}, h_{1 B}, h_{2 A}$, and $h_{2 B}$ of $H^{*}$ such that $h_{i X}$ is irreducible from $P_{i}$ to $X$, where $i$ is either 1 or 2 and $X$ is either $A$ or $B$, and such that $P_{1}$ is not in $h_{2 A}+h_{2 B}$ and $P_{2}$ is not in $h_{1 A}+h_{1 B}$; furthermore, $h_{i A}$ does not have any point in common with $h_{1 B}+h_{2 B}$ not in $p$. There are two mutually exclusive circles $J_{1}$ and $J_{2}$ with centers $P_{1}$ and $P_{2}$, respectively, such that the interior of $J_{1}$ does not intersect $h_{2 A}+h_{2 B}$, and the interior of $J_{2}$ does not intersect $h_{1 A}+h_{1 B}$, and such that there are two mutually exclusive arcs $\gamma_{1}$ and $\gamma_{2}$ irreducible from $P_{1}$ and $P_{2}$, respectively, to an open segment of $\alpha$, such that $\gamma_{1}$ lies, except for its points in $\alpha+$ int. $J_{1}$, in $D$ and does not intersect the interior of $J_{2}$, and $\gamma_{2}$ lies, except for its points in $\alpha+$ int. $J_{2}$, in $D$ and does not intersect the interior of $J_{1}$. Let $C_{1}$ and $C_{2}$ denote the end points of $\gamma_{1}$ and $\gamma_{2}$, respectively, on $\alpha$.

Suppose $A, B, C_{1}$, and $C_{2}$ are in the order $A C_{1} C_{2} B$ on $\alpha$. (The other case can be treated similarly.) Since $\gamma_{1}$ and $\gamma_{2}$ abut on $\alpha$ from the same side, $A C_{1}$ and $\gamma_{2}$ abut on $\gamma_{1}+C_{1} C_{2} B$ from different sides. Thus $A$ and the interior of $J_{2}$ lie in different complementary domains of $h_{1 B}+\gamma_{1}$ $+C_{1} C_{2} B$. But a subcontinuum of $h_{2 A}$ contains $A$, intersects the interior of $J_{2}$, and does not intersect $h_{1 B}+\gamma_{1}+C_{1} C_{2} B$. This is a contradiction.

\section{THEOREM 3. (S) is a continuous curve.}

Proof. Suppose this theorem is false. Then there is a sequence $\mathfrak{W}_{1}, \mathfrak{W}_{2}, \ldots$ of mutually exclusive subcontinua of $\mathbb{B}$ converging to a nondegenerate subcontinuum $\mathbb{S}$ of $\mathbb{S}$, no term of which intersects $\mathbb{S}$. There is a proper subcontinuum $\mathfrak{D}$ of $\mathbb{S}$ which is irreducible between two of its points, $p$ and $q$, such that there exist a positive integer $n$ and an arc $\alpha$ for which $H_{n+1}^{*}+H_{n+2}^{*}+\cdots$ intersects only one complementary domain of $D^{*}$ and $\alpha$ lies, except for its end points, in that complementary domain and is irreducible from $p$ to $q$. Since $G$ is a continuous collection, each element of $D$ is in the boundary of one of the complementary domains $Q^{\prime}$ and $T^{\prime}$ of $D^{*}+\alpha$ having $\alpha$ in their boundaries. Therefore, all of the elements of a subcontinuum $\mathbb{E}$ of $\mathfrak{D}$, which is not a continuum of condensation in $\mathfrak{D}$, lie in the boundary of one of those complementary domains, say $Q$. Since $\mathfrak{D}$ is an irreducible continuum, the boundaries of $Q^{\prime}$ and $T^{\prime}$ intersect each element of $D$. Therefore, there is a dense set of elements of $D$ each containing a point accessible from that one of the domains $Q^{\prime}$ and $T^{\prime}$ which is not $Q$, say $T$. Since $\mathbb{E}$ is not a continuum of condensation in $\mathfrak{D}$, there is an arc $\beta$, lying in $T$ except for its end points, which is ir- 
reducible between some two elements of $E$. Let $\mathfrak{B}$ denote an irreducible subcontinuum of $\xi$ between those two elements. That complementary domain of $B^{*}+\beta$ having $\beta$ on its boundary and not intersecting $\alpha$ does not intersect $Q$. Therefore, $B^{*}$ is a subset of the boundary of a complementary domain of $B^{*}+\beta$ having $\beta$ in its boundary. This contradicts Theorem 2 .

TheOREM 4. Each subarc of (s) contains uncountably many local separating points of $\mathrm{B}$.

Proof. Suppose that a subarc $\mathfrak{S}$ of $\mathbb{S}$ does not contain uncountably many local separating points of $B$. There is an element of $H$ which is

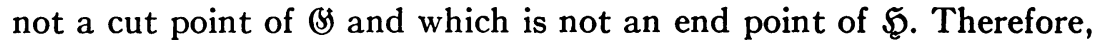
there is an arc in $\mathbb{S}$ having only two points in common with $\mathfrak{S}$. Let $a$ and $b$ denote those points of $\mathfrak{S}$, and let $\mathfrak{R}$ denote the subarc of $\mathfrak{E}$ from $a$ to $b$. No element of $L$ contains elements of $L$ in two of its complementary domains. Let $Z$ denote those elements $g$ of $G$ such that for each element of $L, g$ is in the complementary domain of that element which contains the other elements of $L . L^{*}$ is a subset of $\bar{Z}^{*}$. The argument may be concluded as in the proof of Theorem 3.

THEOREM 5. Each nondegenerate subcontinuum of (S) contains uncountably many local separating points of $\$$ ).

Proof. Each nondegenerate subcontinuum of $B$ is a continuous curve and so contains an arc, which by Theorem 4 contains uncountably many local separating points of $\$ \$ \$$. A list of characterizations of continua having this property is presented on p. 248 of reference [4].

Theorem 6. If G fills up $S$, \& is a dendron.

Proof. A contradiction to Theorem 2 follows immediately the supposition that $\mathbb{B}$ contains a simple closed curve. This theorem and the next also follow easily from various results of this paper and theorems proved either by Moore [6], Vietoris [8], or Whyburn [9].

THEOREм 7. If G fills up $S$ and none of its elements is the boundary of three of its complementary domains, (s) is an arc.

Proof. The decomposition space of a collection of elements of $G$ filling up a complementary domain of an element of $G$ is connected. Hence $\$$ h has no junction point and is an arc.

THEOREM 8. For each dendron $D$, there is a continuous collection $G$ of mutually exclusive decomposable continua filling up $S$ such that (S) is homeomorphic to $D$.

Proof. This result is an immediate corollary of an unpublished 
result announced by $\mathrm{R}$. D. Anderson in the Proceedings of the International Congress (1950). An indication of an argument not dependent on this result is given here.

It is sufficient to show that there exists a continuous collection $G^{\prime}$ of mutually exclusive continua (not necessarily decomposable) filling up a simple closed curve $J$ plus its interior $I$ with each element of $G^{\prime}$ intersecting $J$ and with $\mathcal{S}^{\prime}$ ' homeomorphic to $D$. Let $x_{1}, x_{2}, \cdots$ be a dense collection of cut points of $D$ including all the emanation points of $D$. Proceed inductively. Let $t_{1}$ be a continuum in $J+I$ which is the boundary of each of its complementary domains in $J+I$ and whose complementary domains all intersect $J$ and are in 1-1 correspondence $T_{1}$ with the complementary domains of $x_{1}$ in $D$. For each $i$, let $t_{i}$ be a continuum in the complementary domain of $J+I-\left(t_{1}+t_{2}+\cdots\right.$ $\left.+t_{i-1}\right)$ corresponding under $T_{1}, \cdots, T_{i-1}$ to the complementary domain of $D-\left(x_{1}+\cdots+x_{i-1}\right)$ containing $x_{i}$ with $t_{i}$ satisfying conditions analogous to those imposed on $t_{1}$. By employing rather obvious further conditions on $t_{i}$ with respect to $t_{1}, \cdots, t_{i-1}$ it can be insured that $t_{1}, t_{2}, \cdots$ is a continuous collection admitting the definition of $G^{\prime}$ as required.

\section{BIBLIOGRAPHY}

1. R. D. Anderson, Continuous collections of continuous curves in the plane, Proc. Amer. Math. Soc. vol. 3 (1952) pp. 647-657.

2. - On monotone interior mappings in the plane, Trans. Amer. Math. Soc. vol. 73 (1952) pp. 211-222.

3. Eldon Dyer, Irreducibility of the sum of the elements of a continuous collection of continua, Duke Math. J. vol. 20 (1953) pp. 589-592.

4. O. G. Harrold, Jr., The role of local separating points in certain problems of continuum structure, Lectures in Topology, University of Michigan Conference of 1940, pp. 237-253.

5. R. L. Moore, Foundations of point set theory, Amer. Math. Soc. Colloquium Publications, vol. 13, New York, 1932.

6. - Concerning upper semi-continuous collections, Monatshefte für Mathematik vol. 36 (1929) pp. 81-88.

7. J. H. Roberts, Collections filling a plane, Duke Math. J. vol. 2 (1936) pp. 10-19.

8. L. Vietoris, Über stetige Abbildungen einer Kugelfläche, Proc. Royal Acad. Sciences of Amsterdam vol. 29 (1926) pp. 443-453.

9. G. T. Whyburn, Interior transformations on surfaces, Amer. J. Math. vol. 60 (1938) pp. 477-490.

10. - Analytic topology, Amer. Math. Soc. Colloquium Publications, vol. 28, New York, 1942.

The University of Georgia 